\title{
DR. ROBERTO JARAMILLO URICOECHEA: EL MAESTRO
}

\author{
Sergio Parra Duarte MD*
}

Durante el tiempo de formación y trabajo en el Hospital de San José y la Fundación Universitaria de Ciencias de la Salud tuve la oportunidad de recibir lo que yo llamo "ejemplos de vida" que no son más que experiencias vividas con mis docentes, que les afianzaba su imagen y vocación. De aquellas recuerdo con cariño los ejemplos que el Dr. Roberto Jaramillo impregnó en mí, en su actividad como catedrático, académico y rector. Corría el año 1985 cuando conocí al profesor Jaramillo desde la cátedra de ginecoobstetricia de IX semestre de la Universidad del Rosario. El profesor caminaba por el corredor principal del segundo piso del Hospital de San José con su carrusel de diapositivas bajo su brazo dispuesto a dictarnos clase, al encontrarnos nos dijo "Troncos en lugar de estar aquí mirándome ya deberían estar sentados en el salón, yo nunca llego tarde". El nos sumergía en los conocimientos básicos tanto de la ginecología como de la obstetricia con perfecto conocimiento de cada uno de sus temas y en verdad nunca llegó tarde ni nos dejó esperándole en el aula. El otro ejemplo lo registro en 1989 cuando como residente de primer año de la especialización de ginecoobstetricia, el profesor Jaramillo jefe del programa en la época y líder académico del mismo, nos enseñó a afrontar las vicisitudes propias del entrenamiento con caballerosidad y compromiso, de tal forma que no dudamos en continuar apegados al programa como instructores una vez culminamos el mismo.

A pesar de su arduo trabajo sacaba tiempo para llevarnos de la mano en el desarrollo de una variedad de procedimientos quirúrgicos ginecológicos

\footnotetext{
Rector, Fundación Universitaria de Ciencias de la Salud. Bogotá DC Colombia.
}

algunos simples y otros de alta complejidad, donde apreciamos su destreza y deseo para que alcanzáramos las competencias requeridas en nuestra formación, motivándonos a enfrentar el reto de tratar de imitar su habilidad y elegancia en salas de cirugía, su respeto y afecto en la consulta y su consuelo y buen trato con las pacientes hospitalizadas.

Ya como especialista específicamente recuerdo cómo me delegó el procedimiento quirúrgico "Neovagina con técnica modificada de McIndoe", que realizaba en conjunto con el Dr. Jorge Cantini del Servicio de Cirugía Plástica en el cual era experto y de cuya ejecución se tenía poca experiencia en nuestro medio; en esa ocasión en forma excepcional el profesor recibió y programó dos pacientes con ausencia congénita de vagina, le ayudé en la intervención de la primera paciente y él me dejó operar con su ayuda y supervisión la segunda; una vez culminados los dos casos me dijo: "Negro, de aqui en adelante estos procedimientos quedan bajo su responsabilidad y dirección”.

En 1998 cuando el Dr. Roberto Jaramillo ocupaba el cargo de Decano de la naciente Facultad de Medicina de la Fundación Universitaria de Ciencias de la Salud, me honró al recibirme como su Secretario Académico. Para este momento yo creía que había aprendido suficiente del profesor, pero me demostró que en esta nueva actividad tenía todavía cosas que enseñar; es así como me dió lecciones de redacción, evaluación, comunicación, planeación y de liderazgo de tal forma que me enfocó hacia mi actual proyecto de vida. Como estos tres ejemplos hay otros tantos que viví con él y me permiten reconocer en el Dr. Roberto Jaramillo un verdadero maestro. Pero además de las experiencias comentadas el Dr. Roberto Jaramillo respalda su trasegar académico con su hoja de vida donde se destaca 
como profesor titular de la catedra de Ginecobstetricia de la Pontificia Universidad Javeriana-PUJ y el Colegio Mayor de Nuestra Señora del Rosario, Coordinador del Departamento de Ginecología y Obstetricia del Hospital de San José, Miembro de Número de la Sociedad Colombiana de Gineco Obstetricia, Miembro de Número de la Federación Colombiana de Sociedades de Obstetricia y Ginecología (FECOLSOG), Jefe del Departamento de Ginecología y Obstetricia del Hospital de San José, Miembro de la Federación Internacional de Ginecología y Obstetricia (FIGO), Presidente de la Federación Colombiana de Sociedades de Obstetricia y Ginecología (FECOLSOG), Presidente de la Sociedad de Cirugía de Bogotá, Miembro de Número de la Academia Nacional de Medicina. Maestro de la Ginecología y Obstetricia en Latinoamérica, título conferido por la Federación Latinoamericana de Obstetricia y Ginecología (FLASOG), Decano de la Facultad de Medicina de la Fundación Universitaria de Ciencias de la Salud - FUCS, Miembro honorario de la Asociación Colombiana de Menopausia, Rector de la Fundación Universitaria de Ciencias de la Salud FUCS, Rector Honorario de la Fundación Universitaria de Ciencias de la Salud - FUCS; entre otros tantos títulos que se me quedan en el tintero.

Gracias profesor, lo recordaremos siempre. 